PROCEEDINGS OF THE AMERICAN MATHEMATICAL SOCIETY

Volume 127, Number 7, Pages 1987-1994

S 0002-9939(99)05126-6

Article electronically published on March 17, 1999

\title{
GENERALIZED POWER SYMMETRIC STOCHASTIC MATRICES
}

\author{
R. B. BAPAT, S. K. JAIN, AND K. MANJUNATHA PRASAD
}

(Communicated by Jeffry N. Kahn)

\begin{abstract}
We characterize stochastic matrices $A$ which satisfy the equation $\left(A^{p}\right)^{T}=A^{m}$ where $p<m$ are positive integers.
\end{abstract}

\section{INTRODUCTION}

An $n \times n$ matrix is called stochastic if every entry is nonnegative and each row sum is one. The transpose of the matrix $A$ will be denoted by $A^{T}$. A matrix $A$ is doubly stochastic if both $A$ and $A^{T}$ are stochastic.

Sinkhorn [5] characterized stochastic matrices $A$ which satisfy the condition $A^{T}=A^{p}$, where $p>1$ is a positive integer. Such matrices were called power symmetric in [5]. In this paper we consider a generalization. Call a square matrix $A$ generalized power symmetric if $\left(A^{p}\right)^{T}=A^{m}$, where $p<m$ are positive integers. We give a characterization of generalized power symmetric stochastic matrices, thereby generalizing Sinkhorn's result. The proof makes nontrivial use of the machinery of generalized inverses. In view of the fact that $\left(A^{n}\right)_{i, j}$ represents the probability of an event to change from the state $i$ to the state $j$ in $n$ units of time, the reader may find it interesting to interpret the condition $\left(A^{p}\right)^{T}=A^{m}$ on the matrix $A=\left(A_{i, j}\right)$.

The paper is organized as follows. In the next section, we introduce some definitions and prove several preliminary results. The main results are proved in Section 3 .

\section{Preliminary Results}

If $A$ is an $m \times n$ matrix, then an $n \times m$ matrix $G$ is called a generalized inverse of $A$ if $A G A=A$. If $A$ is a square matrix, then $G$ is the group inverse of $A$ if $A G A=A, G A G=G$ and $A G=G A$. We refer to ([1], [2], [3]) for the background concerning generalized inverses. It is well known that $A$ admits group inverse if and only if $\operatorname{rank}(A)=\operatorname{rank}\left(A^{2}\right)$, in which case the group inverse, denoted by $A^{\#}$, is unique.

Received by the editors October 22, 1997.

1991 Mathematics Subject Classification. Primary 05B20, 15A09, 15A51.

This work was done while the second author was visiting Indian Statistical Institute in November-December 1996 and July-August 1997. He would like to express his thanks for the warm hospitality he enjoyed during his visits as always. Partially supported by Research Challenge Fund, Ohio University. 
If $A$ is an $n \times n$ matrix, then the index of $A$, denoted by index $(A)$, is the least positive integer $k$ such that $\operatorname{rank}\left(A^{k}\right)=\operatorname{rank}\left(A^{k+1}\right)$. Thus $A$ has group inverse if and only if $\operatorname{index}(A)=1$.

If $A$ is an $m \times n$ real matrix, then the $n \times m$ matrix $G$ is called the Moore-Penrose inverse of $A$ if it satisfies $A G A=A, G A G=G,(A G)^{T}=A G,(G A)^{T}=G A$. The Moore-Penrose inverse of $A$, which always exists and is unique, is denoted by $A^{\dagger}$. A real matrix $A$ is said to be an $E P$ matrix if the column spaces of $A$ and $A^{T}$ are identical. We refer to [1] or [3] for elementary properties of $E P$ matrices.

Lemma 1. Let $A$ be a real $n \times n$ matrix and suppose $\left(A^{p}\right)^{T}=A^{m}$, where $p<m$ are positive integers. Then the following assertions are true:

(i) $\operatorname{rank}\left(A^{p}\right)=\operatorname{rank}\left(A^{k}\right), k \geq p$,

(ii) $\operatorname{index}(A) \leq p$,

(iii) $\operatorname{index}\left(A^{p}\right)=1$,

(iv) $A^{p}$ is an EP matrix,

(v) $\left(A^{p}\right)^{\#}=\left(A^{p}\right)^{\dagger}$.

Proof. (i) Clearly, $\operatorname{rank}\left(A^{p}\right) \geq \operatorname{rank}\left(A^{k}\right) \geq \operatorname{rank}\left(A^{m}\right)$ for $p \leq k \leq m$. Since $\left(A^{p}\right)^{T}=A^{m}$, we have

$$
\operatorname{rank}\left(A^{p}\right)=\operatorname{rank}\left(A^{p}\right)^{T}=\operatorname{rank}\left(A^{m}\right)
$$

and thus $\operatorname{rank}\left(A^{p}\right)=\operatorname{rank}\left(A^{k}\right), p \leq k \leq m$. It follows that $\operatorname{rank}\left(A^{p}\right)=$ $\operatorname{rank}\left(A^{k}\right), k \geq p$.

(ii) $\operatorname{By}(\mathrm{i}), \operatorname{rank}\left(A^{p}\right)=\operatorname{rank}\left(A^{p+1}\right)$ and hence $\operatorname{index}(A) \leq p$.

(iii) By (i), $\operatorname{rank}\left(A^{p}\right)=\operatorname{rank}\left(A^{2 p}\right)$ and hence $\operatorname{index}\left(A^{p}\right)=1$.

(iv) Since $\operatorname{rank}\left(A^{p}\right)=\operatorname{rank}\left(A^{m}\right)$, the column space of $A^{p}$ is the same as that of $A^{m}$. Also, since $\left(A^{p}\right)^{T}=A^{m}$, the column space of $A^{m}$ is the same as the row space of $A^{p}$, written as a set of column vectors. Therefore, the column spaces of $A^{p}$ and $\left(A^{p}\right)^{T}$ are identical and $A^{p}$ is an EP matrix.

(v) It is known (see, for example, [3], p. 129) that for an EP matrix the group inverse exists and coincides with the Moore-Penrose inverse.

Lemma 2. Let $A$ be an $n \times n$ matrix such that $\left(A^{p}\right)^{T}=A^{m}$, where $p<m$ are positive integers. Let $\gamma=m-p$. Then $A^{p}=A^{p} A^{i \gamma}\left(A^{i \gamma}\right)^{T}$ for any integer $i \geq 0$.

Proof. We have

$$
\begin{aligned}
A^{p} & =\left(A^{p+\gamma}\right)^{T} \\
& =\left(A^{p}\right)^{T}\left(A^{\gamma}\right)^{T} \\
& =A^{p+\gamma}\left(A^{\gamma}\right)^{T} \\
& =A^{\gamma} A^{p}\left(A^{\gamma}\right)^{T} \\
& =A^{\gamma}\left(A^{\gamma} A^{p}\left(A^{\gamma}\right)^{T}\right)\left(A^{\gamma}\right)^{T} \\
& =A^{2 \gamma} A^{p}\left(A^{2 \gamma}\right)^{T} .
\end{aligned}
$$

Repeating the argument, we get

$$
A^{p}=A^{i \gamma} A^{p}\left(A^{i \gamma}\right)^{T}=A^{p} A^{i \gamma}\left(A^{i \gamma}\right)^{T}
$$

for any $i \geq 0$ and the proof is complete. 
Lemma 3. Let $A$ be a nonnegative $n \times n$ matrix and suppose $\left(A^{p}\right)^{T}=A^{m}$, where $p<m$ are positive integers. Then $\left(A^{p}\right)^{\#}$ exists and is nonnegative.

Proof. By Lemma 1 (iii), index $\left(A^{p}\right)=1$ and therefore $\left(A^{p}\right)^{\#}$ exists. Let $\gamma=m-p$. Setting $i=p$ in Lemma 2, we get

$$
A^{p}=A^{p} A^{p \gamma}\left(A^{p \gamma}\right)^{T}=A^{p} A^{p \gamma} A^{(p+\gamma) \gamma}
$$

in view of $\left(A^{p}\right)^{T}=A^{m}$. Thus, since $\gamma \geq 1$,

$$
A^{p}=A^{2 p} A^{p(\gamma-1)} A^{(p+\gamma) \gamma}
$$

where $A^{o}$ is taken to be the identity matrix. Let $B=A^{p(\gamma-1)} A^{(p+\gamma) \gamma}$. Then it follows from the previous equation that $A^{p} B A^{p}=A^{p}$. Also, since $B$ is a power of $A, A^{p} B=B A^{p}$. Thus $\left(A^{p}\right)^{\#}=B A^{p} B$ is also a power of $A$ and hence is nonnegative.

Lemma 4. Let $A$ be a stochastic $n \times n$ matrix and suppose $\left(A^{p}\right)^{T}=A^{m}$, where $p<m$ are positive integers. Then there exists a permutation matrix $P$ such that $P A P^{T}$ is a direct sum of matrices of the following two types I and II:

I. $C_{11}$ where $C_{11}^{v}=x x^{T}, x>0$ for some positive integer $v$.

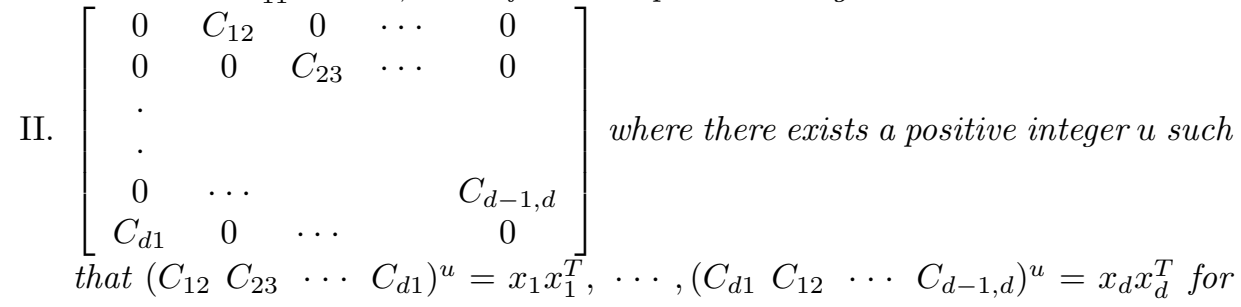
some positive vectors $x_{1}, \cdots, x_{d}$.

Proof. Let $C=A^{p}\left(A^{p}\right)^{\#}$. Since $\left(A^{p}\right)^{\#}=\left(A^{p}\right)^{\dagger}$ by Lemma 1, $C$ is symmetric. By Lemma $3,\left(A^{p}\right)^{\#}$ is nonnegative and hence $C$ is nonnegative. Also, $C$ is clearly idempotent. As observed in the proof of Lemma $3,\left(A^{p}\right)^{\#}$ is a power of $A$ and hence $C$ is also a power of $A$.

The nonnegative roots of symmetric, nonnegative, idempotent matrices have been characterized in [4], Theorem 2. Applying the result to $C$, we get the form of $A$ asserted in the present result. We remark that according to Theorem 2 of [4], a type III summand is also possible along with the two types mentioned above. However, a matrix of this type is necessarily nilpotent and since $A$ is stochastic, such a summand is not possible.

Remark. Using the notation of Lemma 4, it can be verified that the type II summand given there has the property that its $(d u)$ th power is

$$
\left[\begin{array}{cccc}
x_{1} x_{1}^{T} & 0 & \cdots & 0 \\
0 & x_{2} x_{2}^{T} & \cdots & 0 \\
\cdot & & & \\
\cdot & & & \\
0 & 0 & \cdots & x_{d} x_{d}^{T}
\end{array}\right]
$$

This observation will be used in the sequel.

We now introduce some notation. Denote by $J_{m \times n}$ the $m \times n$ matrix with each entry equal to one. If $n_{1}, \cdots, n_{d}$ are positive integers adding up to $n$, then 
$\hat{J}_{\left(n_{1}, \cdots, n_{d}\right)}$ will denote the $n \times n$ matrix

$$
\left[\begin{array}{ccccc}
0 & \frac{1}{n_{2}} J_{n_{1} \times n_{2}} & 0 & \cdots & 0 \\
0 & 0 & \frac{1}{n_{3}} J_{n_{2} \times n_{3}} & \cdots & 0 \\
\cdot & & & & \\
\cdot & & \cdots & & \frac{1}{n_{d}} J_{n_{d-1} \times n_{d}} \\
0 & 0 & \cdots & & 0
\end{array}\right]
$$

where the zero blocks along the diagonal are square, of order $n_{1}, n_{2}, \cdots, n_{d}$, respectively. We remark that if $d=1$, then $\hat{J}_{\left(n_{1}\right)}=\frac{1}{n_{1}} J_{n_{1} \times n_{1}}$.

From now onwards, we make the convention that when we deal with integers $n_{1}, \cdots, n_{d}$, the subscripts of $n$ should be interpreted modulo $d$. Thus, for example, $n_{d+1}=n_{1}, n_{-2}=n_{d-2}$ and so on.

Lemma 5. Let $n_{1}, \cdots, n_{d}$ be positive integers summing to $n$ and let $p<m$ be positive integers. Let $p=\mu \bmod d, m=\mu^{\prime} \bmod d$ where $0 \leq \mu \leq d-1,0 \leq \mu^{\prime} \leq$ $d-1$. Let $S=\hat{J}_{\left(n_{1}, \cdots, n_{d}\right)}$. Then the following conditions are equivalent:

(i) $\left(S^{p}\right)^{T}=S^{m}$;

(ii) (a) $d$ divides both $p$ and $m$, or (b) $d$ divides neither $p$ nor $m, \mu+\mu^{\prime}=d$ and $n_{i}=n_{i+\mu^{\prime}}, \quad 1 \leq i \leq d$

(iii) (a) $d$ divides both $p$ and $m$, or (b) $d$ divides neither $p$ nor $m, \mu+\mu^{\prime}=d$ and $n_{i}=n_{i+\delta}, \quad 1 \leq i \leq d$, where $\delta=(\mu, \mu \prime)$ is the g.c.d. of $\mu$ and $\mu$.

Proof. We first observe that

$$
S^{d}=\left[\begin{array}{cccc}
\frac{1}{n_{1}} J_{n_{1} \times n_{1}} & 0 & \cdots & 0 \\
0 & \frac{1}{n_{2}} J_{n_{2} \times n_{2}} & \cdots & 0 \\
\cdot & & & \\
\cdot & 0 & \cdots & \frac{1}{n_{d}} J_{n_{d} \times n_{d}}
\end{array}\right]
$$

$S^{d} S=S$ and so $S^{d} S^{i}=S^{i}$, for all $i>0$.

Note (i) implies $d$ divides $p$ if and only if $d$ divides $m$. We first prove (i) $\Leftrightarrow(i i)$ :

Assume $d$ divides neither $p$ nor $m$. Let $\left(S^{m}\right)_{i}$ denote the $(i, j)$-block in the partitioning of $S^{\mu}$, in conformity with the partitioning of $S, 1 \leq i, j \leq d$. Similarly $\left(S^{\mu^{\prime}}\right)_{i j}$ will denote the $(i, j)$-block in $S^{\mu^{\prime}}$. A straightforward multiplication involving partitioned matrices shows that

$$
\left(S^{\mu}\right)_{i j}=\left\{\begin{array}{c}
\frac{1}{n_{\mu+i}} J_{n_{i} \times n_{\mu+i}}, \text { if } j=(\mu+i) \bmod d \\
0, \text { otherwise. }
\end{array}\right.
$$

Similarly,

$$
\left(S^{\mu^{\prime}}\right)_{i j}=\left\{\begin{array}{cc}
\frac{1}{n_{\mu^{\prime}+i}} J_{n_{i} \times n_{\mu^{\prime}+i}}, & \text { if } j=\left(\mu^{\prime}+i\right) \bmod d . \\
0, & \text { otherwise. }
\end{array}\right.
$$

Now

$$
\left(S^{\mu}\right)_{i j}^{T}=\left\{\begin{array}{cl}
\frac{1}{n_{\mu+j}} J_{n_{\mu+j} \times n_{j}}, & \text { if } i=(\mu+j) \bmod d \\
0, & \text { otherwise. }
\end{array}\right.
$$

or equivalently,

$$
\left(S^{\mu}\right)_{i j}^{T}=\left\{\begin{array}{cl}
\frac{1}{n_{\mu+j}} J_{n_{\mu+j} \times n_{j}}, & \text { if } j=(i-\mu) \bmod d \\
0, & \text { otherwise. }
\end{array}\right.
$$


Thus $\left(S^{\mu}\right)^{T}=S^{\mu^{\prime}}$ holds if and only if $\mu^{\prime}+i=(i-\mu) \bmod d$ and $n_{i}=n_{\mu^{\prime}+i}$. Since $\left(S^{p}\right)^{T}=S^{m}$ holds if and only if $\left(S^{\mu}\right)^{T}=S^{\mu \prime}$, it follows that under the condition $d$ does not divide $p$ and $d$ does not divide $m$, (i) holds if and only if $\mu+\mu \prime=d$ and $n_{i}=n_{i+\mu \prime}, 1 \leq i \leq d$.

Furthermore, because $d \mid p$ and $d \mid m$ trivially imply $\left(S^{p}\right)^{T}=S^{m}$, the proof of (i) $\Leftrightarrow$ (ii) is completed.

If (ii) holds, then $n_{i}=n_{\mu^{\prime}+i}=n_{d-\mu+i}, \quad 1 \leq i \leq d$. Hence

$$
n_{i}=n_{\alpha \mu^{\prime}-\beta \mu+\beta d+i}=n_{\alpha \mu^{\prime}-\beta \mu+i},
$$

where $\alpha, \beta$ are positive integers, $1 \leq i \leq d$. Choosing $\alpha, \beta$ such that $\delta=\alpha \mu^{\prime}-\beta \mu$, we get $n_{i}=n_{\delta+i}, 1 \leq i \leq d$. This proves (iii).

Conversely, if (iii) holds, then $n_{i}=n_{\mu^{\prime}+i}$ since $\delta$ divides $\mu^{\prime}$, establishing (ii). This completes the proof.

\section{The MAIN RESUlts}

We are now ready to give a characterization of generalized power symmetric stochastic matrices. In the next result we consider matrices of index one.

Theorem 1. Let $A$ be a stochastic $n \times n$ matrix with index $(A)=1$ and let $p<m$ be positive integers. Then $\left(A^{p}\right)^{T}=A^{m}$ if and only if there exists a permutation matrix $P$ such that $P A P^{T}$ is a direct sum of matrices of the following two types I and II:

I. $\quad \frac{1}{k} J_{k \times k}$ for some positive integer $k$;

II. $\quad d \times d$ block partitioned matrices of the form $\hat{J}_{\left(n_{1}, \ldots, n_{d}\right)}$ satisfying (a) $d \mid p$ and $d \mid m$, or (b) $d$ divides neither $p$ nor $m$ such that if $p=\mu(\bmod d), m=$ $\mu \prime(\bmod d)$ where $0 \leq \mu, \mu \prime \leq d-1$ and $\delta=(\mu, \mu \prime)$, then $\mu+\mu \prime=d, n_{i}=n_{i+\delta}$.

Proof. First suppose that $\left(A^{p}\right)^{T}=A^{m}$. By Lemma 4 we conclude that there exists a permutation matrix $P$ such that $P A P^{T}$ is a direct sum of matrices of types I, II given in Lemma 4.

Let $S=C_{11}$ be a summand of type I so that $C_{11}^{v}=x x^{T}, x>0$ for some positive integer $v$. Since $i n d e x\left(C_{11}\right)=1$, it follows that $\operatorname{rank}\left(C_{11}\right)=\operatorname{rank}\left(C_{11}^{v}\right)=1$. Suppose $C_{11}=\tilde{x} \tilde{y}^{T}$ for some $\tilde{x}>0, \tilde{y}>0$. Now using $\left(C_{11}^{p}\right)^{T}=C_{11}^{m}$, it can be shown that $C_{11}$ is symmetric and hence doubly stochastic. It is well known that a positive, rank one doubly stochastic matrix must be $\frac{1}{k} J_{k \times k}$ for some $k$.

Now suppose $S$ is a summand of type II and let

$$
S=\left[\begin{array}{ccccc}
0 & C_{12} & 0 & \cdots & 0 \\
0 & 0 & C_{23} & \cdots & 0 \\
\cdot & & & & \\
\cdot & & & & C_{d-1, d} \\
0 & 0 & \cdots & & 0 \\
C_{d 1} & 0 & \cdots & & 0
\end{array}\right]
$$

where the zero blocks on the diagonal are square of order $n_{1}, \cdots, n_{d}$, respectively. As observed in the remark following Lemma 4, there exists a positive integer $u$ such that $S^{d u}$ is a direct sum of $d$ positive, symmetric, idempotent, rank one matrices.

We first claim that $\operatorname{rank}\left(C_{12}\right)=\operatorname{rank}\left(C_{23}\right)=\cdots=\operatorname{rank}\left(C_{d 1}\right)=1$. For otherwise, $\operatorname{rank}(S)>d$. However, $\operatorname{rank}\left(S^{d u}\right)=d$ and since $\operatorname{index}(S)=1, \operatorname{rank}(S)=$ $\operatorname{rank}\left(S^{d u}\right)$, giving a contradiction. Therefore, the claim is proved. We let $C_{12}=$ 
$x_{1} y_{2}^{T}, C_{23}=x_{2} y_{3}^{T}, \cdots, C_{d 1}=x_{d} y_{1}^{T}$, where $x_{i}, y_{i}$ are positive vectors. Since $C_{12}, C_{23}, \cdots, C_{d 1}$ have rank one, we may choose $x_{i}=J_{n_{i} \times 1}, 1 \leq i \leq d$.

In view of the description of $S^{d u}$ given earlier, we may write

$$
\left(C_{12} C_{23} \cdots C_{d 1}\right)^{u}=x x^{T}
$$

for some positive vector $x$. Thus

$$
\left(x_{1} y_{2}^{T} x_{2} y_{3}^{T} \cdots x_{d} y_{1}^{T}\right)^{u}=x x^{T}
$$

and hence $\lambda x_{1} y_{1}^{T}=x x^{T}$ for some $\lambda>0$. It follows that $y_{1}=\gamma_{1} J_{n_{1} \times 1}$ for some $\gamma_{1}>0$. Similarly we conclude that $y_{i}=\gamma_{i} J_{n_{i} \times 1}, \gamma_{i}>0,1 \leq i \leq d$. Now, interpreting subscripts modulo $d$ as usual, we have

$$
\begin{aligned}
C_{i, i+1} & =x_{i} y_{i+1}^{T} \\
& =J_{n_{i} \times 1}\left(\gamma_{i+1} J_{n_{i+1} \times 1}\right)^{T} \\
& =\gamma_{i+1} J_{n_{i} \times n_{i+1}} \\
& =\frac{1}{n_{i+1}} J_{n_{i} \times n_{i+1}},
\end{aligned}
$$

since $C_{i, i+1}$ has row sums one, $1 \leq i \leq d$. The remaining assertions in (ii) follow from Lemma 5.

Conversely, it is clear that if $S$ is a summand of type I or II, then $\left(S^{p}\right)^{T}=S^{m}$. This completes the proof.

Corollary 1. Let $A$ be an $n \times n$ stochastic matrix of index one and suppose $\left(A^{p}\right)^{T}=$ $A^{m}$ where $p<m$ are positive integers with $(p, m)=1$. Then there exists a permutation matrix $P$ such that $P A P^{T}$ is a direct sum of matrices of the following types $I$ and $I I$ :

I. $\frac{1}{k} J_{k \times k}$ for some positive integer $k$;

II. $\hat{J}_{(\ell, \ldots, \ell)}$ for some positive integer $\ell$, occurring $d$ times and $d$ divides $p+m$.

Proof. By Theorem 1 there exists a permutation matrix $P$ such that $P A P^{T}$ is a direct sum of matrices of types I, II given in Theorem 1. Consider a summand of type II, say $\hat{J}_{\left(n_{1}, \cdots, n_{d}\right)}$. Let $\mu=p \bmod d, \mu^{\prime}=m \bmod d$. Then by Theorem 1 , $\mu+\mu^{\prime}=d$ and $n_{i}=n_{i+\delta}$, where $\delta$ is the g.c.d. of $\mu, \mu^{\prime}$. Since $(p, m)=1$ and $\mu+\mu^{\prime}=d$, it follows that $\delta=1$. Thus $n_{1}=n_{2}=\cdots=n_{d}=\ell$, say. Thus the summand equals $\hat{J}_{(\ell, \ldots, \ell)}$. Also, $\mu+\mu^{\prime}=d$ implies that $p+m=0 \bmod d$ and thus $d$ divides $p+m$.

We now derive the main result in [5].

Corollary 2. Let $A$ be an $n \times n$ stochastic matrix and suppose $A^{T}=A^{m}$ for some positive integer $m>1$. Then there exists a permutation matrix $P$ such that $P A P^{T}$ is a direct sum of matrices of the following types:

(1) $\frac{1}{k} J_{k \times k}$ for some positive integer $k$;

(2) $\hat{J}_{(\ell, \ldots, \ell)}$ for some positive integer $\ell$, where $\ell$ occurs $d$ times and $d$ divides $m+1$.

Proof. By Lemma 1, index $(A)=1$. Since the g.c.d. of $1, m$ is 1 , by Corollary 1 there exists a permutation matrix $P$ such that $P A P^{T}$ is a direct sum of matrices of types given in Corollary 1 . The rest of the proof follows easily. 
Our next objective is to describe a stochastic matrix $A$, not necessarily of index one, which satisfies $\left(A^{p}\right)^{T}=A^{m}$ for positive integers $p<m$.

If $A$ is an $n \times n$ matrix, then recall that $A$ can be expressed as $A=C_{A}+N_{A}$ where $C_{A}$, the core part of $A$, is of index one, $N_{A}$ is nilpotent and $C_{A} N_{A}=N_{A} C_{A}=0$. This is referred to as the core-nilpotent decomposition of $A$. We refer to [2] for basic properties of this decomposition.

Lemma 6. Let $A$ be a stochastic $n \times n$ matrix such that $\left(A^{p}\right)^{T}=A^{m}$, where $p<m$ are positive integers. Let $A=C_{A}+N_{A}$ be the core-nilpotent decomposition. Then $C_{A}$ is nonnegative and stochastic.

Proof. By Lemma $1, \operatorname{index}(A) \leq p$. Then $A^{p}=C_{A}^{p}$. Since $i n d e x\left(C_{A}\right)=1$, we can write $C_{A}=C_{A}^{p} X$ for some matrix $X$. Now

$$
\begin{aligned}
A^{p}\left(A^{p}\right)^{\#} C_{A} & =A^{p}\left(A^{p}\right)^{\#} C_{A}^{p} X \\
& =A^{p}\left(A^{p}\right)^{\#} A^{p} X \\
& =A^{p} X \\
& =C_{A}^{p} X \\
& =C_{A}
\end{aligned}
$$

and then

$$
\begin{aligned}
A^{p}\left(A^{p}\right)^{\#} A & =A^{p}\left(A^{p}\right)^{\#}\left(C_{A}+N_{A}\right) \\
& =C_{A}+A^{p}\left(A^{p}\right)^{\#} N_{A} \\
& =C_{A}+\left(A^{p}\right)^{\#} C_{A}^{p} N_{A} \\
& =C_{A} .
\end{aligned}
$$

Since $\left(A^{p}\right)^{\#}$ is a power of $A$, as observed in the proof of Lemma 3 , it follows that $C_{A}$ is also a power of $A$. Thus $C_{A}$ is nonnegative and stochastic.

Theorem 2. Let $A$ be an $n \times n$ stochastic matrix. Then $\left(A^{p}\right)^{T}=A^{m}$, where $p<m$ are positive integers if and only if there exists a permutation matrix $P$ such that $P A P^{T}$ is a direct sum of matrices $C_{i i}+N_{i i}, 1 \leq i \leq k$, where

(1) $C_{i i}$ are stochastic matrices of index $1, N_{i i}$ are nilpotent matrices of index $\leq p$ with sum of entries of each row as zero, $C_{i i} N_{i i}=0=N_{i i} C_{i i}, 1 \leq i \leq k$, and

(2) Each $C_{i i}$ is a direct sum of matrices of types (I) and (II) as described in Theorem 1.

Proof. 'Only if' part: By Lemma 1, index $(A) \leq p$ and hence $A^{p}=C_{A}^{p}, A^{m}=C_{A}^{m}$. Thus $\left(C_{A}^{p}\right)^{T}=C_{A}^{m}$. Since index $\left(C_{A}\right)=1$ and by Lemma $6 C_{A}$ is stochastic, Theorem 1 yields that there exists a permutation matrix $P$ such that $P C_{A} P^{T}$ is a direct sum of matrices of types I, II as given in Theorem 1.

Let

$$
P A P^{T}=\left[A_{i j}\right], P C_{A} P^{T}=\left[\left(C_{A}\right)_{i j}\right], \quad P N_{A} P^{T}=\left[\left(N_{A}\right)_{i j}\right]
$$

be compatible partitions. Note that $\left(C_{A}\right)_{i j}=0$ if $i \neq j$. Since $A_{i j}=\left(C_{A}\right)_{i j}+$ $\left(N_{A}\right)_{i j}$, we get that $\left(N_{A}\right)_{i j} \geq 0$ for $i \neq j$. However, since $C_{A} N_{A}=0$, we have $\left(C_{A}\right)_{i i}\left(N_{A}\right)_{i j}=0, i \neq j$. It follows from the description of $\left(C_{A}\right)_{i i}$, in Theorem 1 and the remark following Lemma 4 that for some positive integer $s,\left(C_{A}\right)_{i i}^{s}$ has 
positive diagonal entries. This, along with $\left(C_{A}\right)_{i i}\left(N_{A}\right)_{i j}=0$, and $\left(N_{A}\right)_{i j} \geq 0$ for $i \neq j$, yields $\left(N_{A}\right)_{i j}=0$. Thus $A_{i j}=0, i \neq j$. Setting $C_{i i}=\left(C_{A}\right)_{i i}, N_{i i}=\left(N_{A}\right)_{i i}$ for all $i$, the result follows. The 'if part' is straightforward.

We conclude with an example. Let

$$
A=\left[\begin{array}{cccc}
0 & 0 & \frac{1}{2} & \frac{1}{2} \\
0 & 0 & \frac{1}{2} & \frac{1}{2} \\
\frac{1}{3} & \frac{2}{3} & 0 & 0 \\
\frac{2}{3} & \frac{1}{3} & 0 & 0
\end{array}\right]
$$

Then $A$ is stochastic and $\left(A^{2}\right)^{T}=A^{4}$. The core-nilpotent decomposition is given by $A=C_{A}+N_{A}$, where

$$
C_{A}=\left[\begin{array}{cccc}
0 & 0 & \frac{1}{2} & \frac{1}{2} \\
0 & 0 & \frac{1}{2} & \frac{1}{2} \\
\frac{1}{2} & \frac{1}{2} & 0 & 0 \\
\frac{1}{2} & \frac{1}{2} & 0 & 0
\end{array}\right] \quad, \quad N_{A}=\left[\begin{array}{cccc}
0 & 0 & 0 & 0 \\
0 & 0 & 0 & 0 \\
\frac{-1}{6} & \frac{1}{6} & 0 & 0 \\
\frac{1}{6} & \frac{-1}{6} & 0 & 0
\end{array}\right]
$$

Thus $C_{A}$ is stochastic and is of type II as described in Theorem 2. This example also shows that $N_{A}$ need not be nonnegative.

\section{ACKNOWLEDGEMENT}

The authors would like to thank the referee for helpful suggestions and comments.

\section{REFERENCES}

[1] A. Ben-Israel and T.N.E. Greville, Generalized Matrix Inverses: Theory and Applications, Wiley, NY, 1973. MR 53:469

[2] A. Berman and R.J. Plemmons, Nonnegative Matrices in the Mathematical Sciences, 2nd edition, SIAM, 1995. MR 95e:15013

[3] S. L. Campbell and C.D. Meyer, Jr., Generalized Inverses of Linear Transformations, Pitman, 1979. MR 80d:15003

[4] S. K. Jain, V.K. Goel and Edward K. Kwak, Nonnegative mth roots of nonnegative Osymmetric idempotent matrices, Linear Algebra Appl. 23:37-51 (1979). MR 80m:15002

[5] Richard Sinkhorn, Power symmetric stochastic matrices, Linear Algebra Appl. 40:225-228 (1981). MR 82j: 15017

Department of Mathematics, Indian Statistical Institute, 7 S.J.S. Sansanwal Marg, New Delhi 110016, India

E-mail address: rbb@isidl.isid.ac.in

Department of Mathematics, Ohio University, Athens, Ohio 45701

E-mail address: jain@math.ohiou.edu

Department of Mathematics, Manipal Institute of Technology, Gangtok, Sikkim, 737102 INDIA 